Utah State University

DigitalCommons@USU

Space Dynamics Lab Publications

Space Dynamics Lab

$1-1-2006$

\title{
SOFIE Instrument Model and Performance Comparison
}

\author{
Scott Hansen \\ Andrew Shumway \\ Chad Fish \\ Jim Peterson \\ Peter Mace \\ James Cook
}

See next page for additional authors

Follow this and additional works at: https://digitalcommons.usu.edu/sdl_pubs

\section{Recommended Citation}

Hansen, Scott; Shumway, Andrew; Fish, Chad; Peterson, Jim; Mace, Peter; Cook, James; Nelsen, Joil; Hooper, Dale; Young, Quinn; Wassom, Steve; Kemp, John; Gordley, Larry; and Hervig, Mark, "SOFIE Instrument Model and Performance Comparison" (2006). Space Dynamics Lab Publications. Paper 58.

https://digitalcommons.usu.edu/sdl_pubs/58

This Article is brought to you for free and open access by the Space Dynamics Lab at DigitalCommons@USU. It has been accepted for inclusion in Space Dynamics Lab Publications by an authorized administrator of DigitalCommons@USU. For more information, please contact digitalcommons@usu.edu.

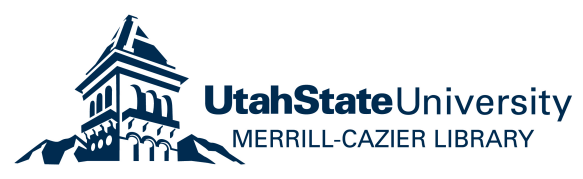




\section{Authors}

Scott Hansen, Andrew Shumway, Chad Fish, Jim Peterson, Peter Mace, James Cook, Joil Nelsen, Dale Hooper, Quinn Young, Steve Wassom, John Kemp, Larry Gordley, and Mark Hervig 


\author{
SOFIE Instrument Model and Performance Comparison \\ Scott Hansen ${ }^{1}$,Andrew Shumway ${ }^{1}$, Chad Fish ${ }^{1}$, Jim Peterson ${ }^{1}$, Peter Mace ${ }^{1}$, James Cook ${ }^{1}$, Joel \\ Nelsen ${ }^{1}$, Dale Hooper ${ }^{1}$, Quinn Young ${ }^{1}$, Steve Wassom ${ }^{1}$, John Kemp ${ }^{1}$, Larry Gordley ${ }^{2}$, Mark Hervig ${ }^{3}$ \\ ${ }^{1}$ Space Dynamics Laboratory/Utah State University \\ 1695 North Research Park Way, North Logan, UT 84341 \\ ${ }^{2}$ GATS, Inc. \\ 11864 Canon Blvd., Newport News, VA 23606 \\ ${ }^{3}$ GATS, Inc. \\ P.O. Box 449, Driggs, ID 83422
}

\begin{abstract}
Space Dynamics Laboratory (SDL), in partnership with GATS, Inc., designed, built, and calibrated an instrument to conduct the Solar Occultation for Ice Experiment (SOFIE). SOFIE is the primary infrared sensor in the NASA Aeronomy of Ice in the Mesosphere (AIM) instrument suite. AIM's mission is to study polar mesospheric clouds (PMCs). SOFIE will make measurements in 16 separate spectral bands, arranged in 8 pairs between 0.29 and $5.3 \mu \mathrm{m}$. Each band pair will provide differential absorption limb-path transmission profiles for an atmospheric component of interest, by observing the sun through the limb of the atmosphere during solar occultation as AIM orbits Earth. A fast steering mirror and imaging sun sensor coaligned with the detectors will track the sun during occultation events and maintain stable alignment of the Sun on the detectors.

This paper outlines the instrument specifications and resulting design. The success of the design process followed at SDL is illustrated by comparison of instrument model calculations to calibration results, and lessons learned during the SOFIE program are discussed. Relative spectral response predictions based on component measurements are compared to end-to-end spectral response measurements. Field-of-view measurements are compared to design expectations, and radiometric predictions are compared to results from blackbody and solar measurements. Measurements of SOFIE detector response non-linearity are presented, and compared to expectations based on simple detector models.
\end{abstract}

Keywords: SOFIE, AIM, fabrication, integration, test, calibration, solar, infrared

\title{
1. INTRODUCTION
}

SOFIE (Solar Occultation for Ice Experiment) is the primary infrared sensor in the Aeronomy of Ice in the Mesosphere (AIM) suite of instruments. The SOFIE instrument design is based on the highly successful Upper Atmosphere Research Satellite (UARS) Halogen Occultation Experiment (HALOE) instrument. SOFIE will perform satellite solar occultation measurements to obtain limb-path broadband transmission profiles, which will be used to determine profiles of temperature/pressure, mixing ratio profiles of five gaseous species $(\mathrm{O} 3, \mathrm{H} 2 \mathrm{O}, \mathrm{CO} 2, \mathrm{CH} 4$, and $\mathrm{NO})$, and polar mesospheric cloud (PMC) particle extinction.

The instrument will use the differential absorption radiometry approach, with eight channel pairs covering wavelengths $(\lambda)$ from 0.29 to $5.32 \mu \mathrm{m}$. In this technique, specific gases are targeted by measuring solar intensity in two wavelength regions, one where the gas is strongly absorbing and an adjacent region where the gas is weakly absorbing. Measuring the difference between the strong and weak band signals has numerous advantages, including the reduction of undesired instrumental effects, and the reduction of atmospheric interference. Six SOFIE channels are designed to measure gaseous signals, and two are dedicated to particle measurements. Measurements in two $\mathrm{CO}_{2}$ bands will be used to simultaneously retrieve temperature and $\mathrm{CO}_{2}$ mixing ratio. In addition to the main science channels, SOFIE includes an imaging sun sensor for pointing control.

Infrared Spaceborne Remote Sensing XIV, edited by Marija Strojnik, Proc. of SPIE Vol. 6297, 62970P, (2006) - 0277-786X/06/\$15 - doi: 10.1117/12.692859 


\section{INSTRUMENT REQUIREMENTS AND DESIGN}

\subsection{Science Overview}

The primary science objective of the NASA AIM mission is to study PMCs, also known as noctilucent clouds. Around $83 \mathrm{~km}$ in altitude, these are the highest clouds in our atmosphere and form poleward of $50^{\circ}$ latitude in both hemispheres during summer months. Observations to date indicate they are increasing in number and brightness, and are being found closer to the equator. It is not understood how these clouds are formed, but hypotheses are being made that these clouds may be strongly correlated to global climate stability. Questions are being asked about the relationship between these clouds and global temperature, atmospheric dynamics, and chemistry.

The SOFIE instrument is specifically designed to measure gasses, temperature, and particles in this cloud layer. SOFIE uses the solar occultation method to measure $\mathrm{H}_{2} \mathrm{O}, \mathrm{CO}_{2}, \mathrm{O}_{3}, \mathrm{CH}_{4}$, and $\mathrm{NO}$, along with temperature and particle extinction at 16 wavelengths.

\subsection{Instrument Requirements}

The AIM satellite will be launched on a Pegasus rocket into a $600-650 \mathrm{~km}$ polar Sun-synchronous orbit where it is required to operate for 25 months. SOFIE will perform two solar occultation measurements per orbit, creating a data volume of $168 \mathrm{Mbits}$ per day. The steering mirror with a $2^{\circ} \mathrm{X} 2^{\circ}$ degree field of regard allows SOFIE to locate and center on the Sun. During a solar occultation, SOFIE stares through a 1.8 X 6 arc-minute (vertical X horizontal) field at the center of the Sun. A sun sensor tracking system references the top edge of the Sun to track this position within 15 arc-seconds. With a vertical spatial resolution $<3 \mathrm{~km}$, SOFIE is designed to track the Sun from $315 \mathrm{~km}$ down to the hard edge of the Earth.

Sixteen bands pair up to form 8 channels measuring $\mathrm{H}_{2} \mathrm{O}, \mathrm{CO}_{2}, \mathrm{O}_{3}, \mathrm{CH}_{4}$, and $\mathrm{NO}$ along with particle channels as shown in Table 1. The shortest wavelength bands use silicon carbide and germanium detectors, while the longer wavelength bands use mercury-cadmium-telluride detectors. These bands are spectrally grouped in channel pairs consisting of a strongly and weakly absorbing band for each target gas or particles. When a target gas is measured, the strongly absorbing band detects energy in a band that is mostly attenuated by the gas, so that the detector signal drops sharply, while the weakly absorbing band changes much less dramatically. A third signal is the difference between the strong and weak bands, which is amplified to preserve high resolution information that is not recoverable by ground postprocessing of the strong and weak band signals alone.

Table 1: SOFIE Channel Requirements Overview

\begin{tabular}{|c|c|c|c|c|}
\hline Channel & Band / Target & $\begin{array}{c}\text { Center } \lambda \\
(\mu \mathrm{m})\end{array}$ & Detector & Signal / Noise \\
\hline \multirow{2}{*}{1} & $1 / \mathrm{O}_{3}$ strong & 0.290 & \multirow{2}{*}{ SiC, PV } & \multirow{2}{*}{$1.0 \times 10^{4}$} \\
\hline & $2 / \mathrm{O}_{3}$ weak & 0.328 & & \\
\hline \multirow{2}{*}{2} & 3 / Particles strong & 0.862 & \multirow{2}{*}{$\mathrm{Ge}, \mathrm{PV}$} & \multirow{2}{*}{$1.0 \times 10^{6}$} \\
\hline & 4 / Particles weak & 1.031 & & \\
\hline \multirow[t]{2}{*}{3} & $5 / \mathrm{H}_{2} \mathrm{O}$ weak & 2.45 & \multirow{2}{*}{$\mathrm{HgCdTe}, \mathrm{PC}$} & \multirow[t]{2}{*}{$2.5 \times 10^{4}$} \\
\hline & $6 / \mathrm{H}_{2} \mathrm{O}$ strong & 2.60 & & \\
\hline \multirow[t]{2}{*}{4} & $7 / \mathrm{CO}_{2}$ strong & 2.77 & \multirow{2}{*}{$\mathrm{HgCdTe}, \mathrm{PC}$} & \multirow[t]{2}{*}{$3.0 \times 10^{5}$} \\
\hline & $8 / \mathrm{CO}_{2}$ weak & 2.94 & & \\
\hline \multirow[t]{2}{*}{5} & 9 / Particles strong & 3.06 & \multirow{2}{*}{$\mathrm{HgCdTe}, \mathrm{PC}$} & \multirow[t]{2}{*}{$1.0 \times 10^{5}$} \\
\hline & 10 / Particles weak & 3.19 & & \\
\hline \multirow[t]{2}{*}{6} & $11 / \mathrm{CH}_{4}$ strong & 3.37 & \multirow[t]{2}{*}{ HgCdTe, PC } & \multirow[t]{2}{*}{$4.0 \times 10^{5}$} \\
\hline & $12 / \mathrm{CH}_{4}$ weak & 3.51 & & \\
\hline
\end{tabular}




\begin{tabular}{|c|c|c|c|c|}
\hline Channel & Band / Target & $\begin{array}{c}\text { Center } \lambda \\
(\mu \mathbf{m})\end{array}$ & Detector & Signal / Noise \\
\hline \multirow{2}{*}{7} & $13 / \mathrm{CO}_{2}$ strong & 4.25 & \multirow{2}{*}{ HgCdTe, PC } & $4.0 \times 10^{5}$ \\
\cline { 2 - 3 } & $14 / \mathrm{CO}_{2}$ weak & 4.63 & & \multirow{2}{*}{ HgCdTe, PC } \\
\hline \multirow{2}{*}{8} & $15 /$ NO weak & 4.98 & $3.0 \times 10^{5}$ \\
\cline { 2 - 3 } & $16 /$ NO strong & 5.32 & & \\
\hline
\end{tabular}

The high signal to noise ratios specified in Table 1 were driving requirements in choosing a $10 \mathrm{~cm}$ diameter telescope and large detector gains in the electronics. The $\Delta \mathrm{V}$ signal dynamic range requirement was $2^{13}$ counts. The maximum acceptable nonlinearity was specified to be $0.5 \%$ for the direct detector signals (strong and weak signals) and $1 \%$ for the $\Delta \mathrm{V}$ signals.

\section{RADIOMETRIC SIMULATION PARAMETERS}

Detailed theoretical models of the SOFIE science detectors and steering mirror focal plane were implemented to understand instrument performance. These tools were used to understand instrument response during component testing and integration, and to develop calibration plans. Parameters used in the radiometric simulation models are presented here.

\subsection{Science Detectors}

A detailed radiometric model for the SOFIE science detectors was implemented in MathCAD (Mathsoft Engineering and Education, Inc.). A listing of SOFIE parameters used in the model is shown in Table 2. Parameters unique to individual SOFIE detectors are shown in Table 3.

Table 2: SOFIE Radiometric Model Parameters

\begin{tabular}{|c|c|}
\hline Parameter & Value \\
\hline Entrance Pupil Area & $74 \mathrm{~cm}^{2}$ \\
\hline Detector FOV & $1.8 \times 6.0 \mathrm{arcmin}$ \\
\hline Detector Area & $0.01 \mathrm{~cm}^{2}$ \\
\hline Electrical Noise Bandwidth & $3.14 \mathrm{~Hz}$ \\
\hline Chopper Modulation Loss & 0.406 \\
\hline
\end{tabular}

Table 3: SOFIE Detector Parameters

\begin{tabular}{|c|c|c|}
\hline Band / Target & $\begin{array}{c}\text { Detectivity }\left(\mathbf{D}^{*}\right) \\
(\mathbf{c m} \sqrt{\mathbf{H z}} / \mathbf{W})\end{array}$ & $\begin{array}{c}\text { Incident Solar Flux } \\
(\mathbf{m W})\end{array}$ \\
\hline $1 / \mathrm{O}_{3}$ strong & $1.8 \times 10^{12}$ & $3.888 \times 10^{-2}$ \\
\hline $2 / \mathrm{O}_{3}$ weak & $1.8 \times 10^{12}$ & $1.554 \times 10^{-1}$ \\
\hline 3 / Particles strong & $7.5 \times 10^{11}$ & $2.606 \times 10^{-1}$ \\
\hline $4 /$ Particles weak & $4.1 \times 10^{11}$ & $5.941 \times 10^{-1}$ \\
\hline $5 / \mathrm{H}_{2} \mathrm{O}$ weak & $1.4 \times 10^{10}$ & $7.035 \times 10^{-2}$ \\
\hline $6 / \mathrm{H}_{2} \mathrm{O}$ strong & $1.6 \times 10^{10}$ & $5.670 \times 10^{-2}$ \\
\hline $7 / \mathrm{CO}_{2}$ strong & $4.6 \times 10^{10}$ & $4.735 \times 10^{-2}$ \\
\hline
\end{tabular}




\begin{tabular}{|c|c|c|}
\hline Band / Target & $\begin{array}{c}\text { Detectivity }\left(\mathbf{D}^{*}\right) \\
(\mathbf{c m} \sqrt{\mathbf{H z}} / \mathbf{W})\end{array}$ & $\begin{array}{c}\text { Incident Solar Flux } \\
(\mathbf{m W})\end{array}$ \\
\hline $8 / \mathrm{CO}_{2}$ weak & $4.8 \times 10^{10}$ & $4.200 \times 10^{-2}$ \\
\hline $9 /$ Particles strong & $1.8 \times 10^{10}$ & $3.232 \times 10^{-2}$ \\
\hline $10 /$ Particles weak & $2.0 \times 10^{10}$ & $3.216 \times 10^{-2}$ \\
\hline $11 / \mathrm{CH}_{4}$ strong & $1.9 \times 10^{10}$ & $3.304 \times 10^{-2}$ \\
\hline $12 / \mathrm{CH}_{4}$ weak & $2.4 \times 10^{10}$ & $2.629 \times 10^{-2}$ \\
\hline $13 / \mathrm{CO}_{2}$ strong & $5.1 \times 10^{10}$ & $2.920 \times 10^{-2}$ \\
\hline $14 / \mathrm{CO}_{2}$ weak & $5.6 \times 10^{10}$ & $1.490 \times 10^{-2}$ \\
\hline $15 / \mathrm{NO}^{10}$ weak & $4.5 \times 10^{10}$ & $8.081 \times 10^{-3}$ \\
\hline $16 / \mathrm{NO}$ strong & $3.4 \times 10^{10}$ & $1.159 \times 10^{-2}$ \\
\hline
\end{tabular}

\subsection{Steering Mirror Focal Plane}

The sun sensor and steering mirror system is very complex and required much early testing to validate both the electronics and software. Parameters used to implement a detailed radiometric model for the sun sensor are listed in Table 4.

Table 4: SOFIE Steering Mirror Model Parameters

\begin{tabular}{|c|c|}
\hline Parameter & Value \\
\hline Bandpass Filter Band Edges & $694-706 \mathrm{~nm}$ \\
\hline Bandpass Filter Transmittance & 0.4 \\
\hline Neutral Density Filter & ND 1.6 \\
\hline Entrance Pupil Area & $0.785 \mathrm{~cm}^{2}$ \\
\hline Pixel FOV & $0.13 \times 0.13 \mathrm{arcmin}$ \\
\hline Pixel Area & $2.25 \times 10^{-6} \mathrm{~cm}^{2}$ \\
\hline Telescope Transmittance & 0.922 \\
\hline Effective Quantum Efficiency & $20 \%$ \\
\hline Well capacity & $135,000 \mathrm{e}^{-}$ \\
\hline
\end{tabular}

\subsection{Source Parameters}

Source parameters used in both science detector and steering mirror focal plane radiometric models are presented here. Sources used for the SOFIE science bands include a solar emulator blackbody (SEBB), and the sun observed through the atmosphere. Sources used to test the SOFIE steering mirror focal lane included an incandescent lamp in addition to the sun.

\subsubsection{Solar Emulator Blackbody}

A basic parameter in the SOFIE science radiometric model is blackbody radiance, which depends on accurate temperature knowledge of the SEBB cavity. During SOFIE calibration, an Electro-Optical Industries model 156A blackbody was used to stimulate the instrument. This blackbody was calibrated at SDL using an optical pyrometer to observe the blackbody cavity and correlate cavity effective temperature to controller dial position. This relationship is plotted in Figure 1. The calibration curve shown was used to generate response predictions used for system gain adjustment and calibration test planning.

The blackbody cavity failed during SOFIE calibration operations following the first blackbody calibration in September 2005. After the blackbody cavity was replaced, a second blackbody calibration was performed in February 2006. During 
this test, multiple temperature measurements were made to estimate uncertainty. The error bars shown in the plot indicate the standard deviation of these multiple temperature measurements. These measurements showed that blackbody performance following the cavity rebuild remained consistent with that observed before the cavity failed.

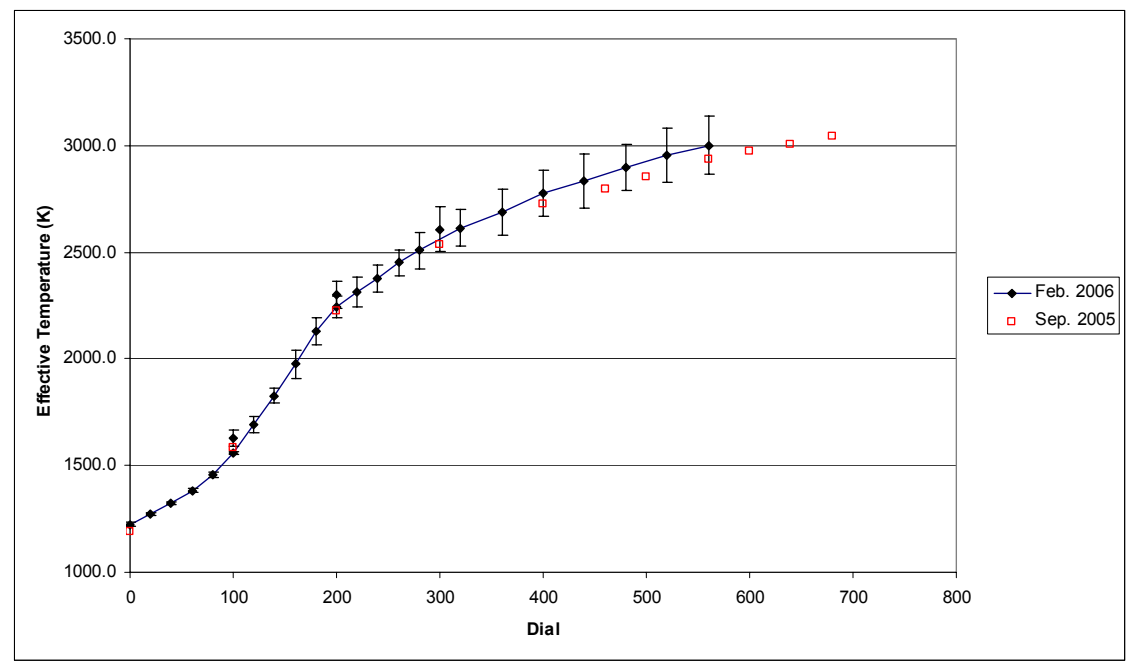

Figure 1. Solar Emulator Blackbody Calibration Curve

\subsubsection{Solar Observation}

Solar observations were used to test performance of both the science bands and the steering mirror focal plane. Solar radiance data used for model predictions are shown in Figure 2, with a $6000 \mathrm{~K}$ blackbody curve shown for comparison.

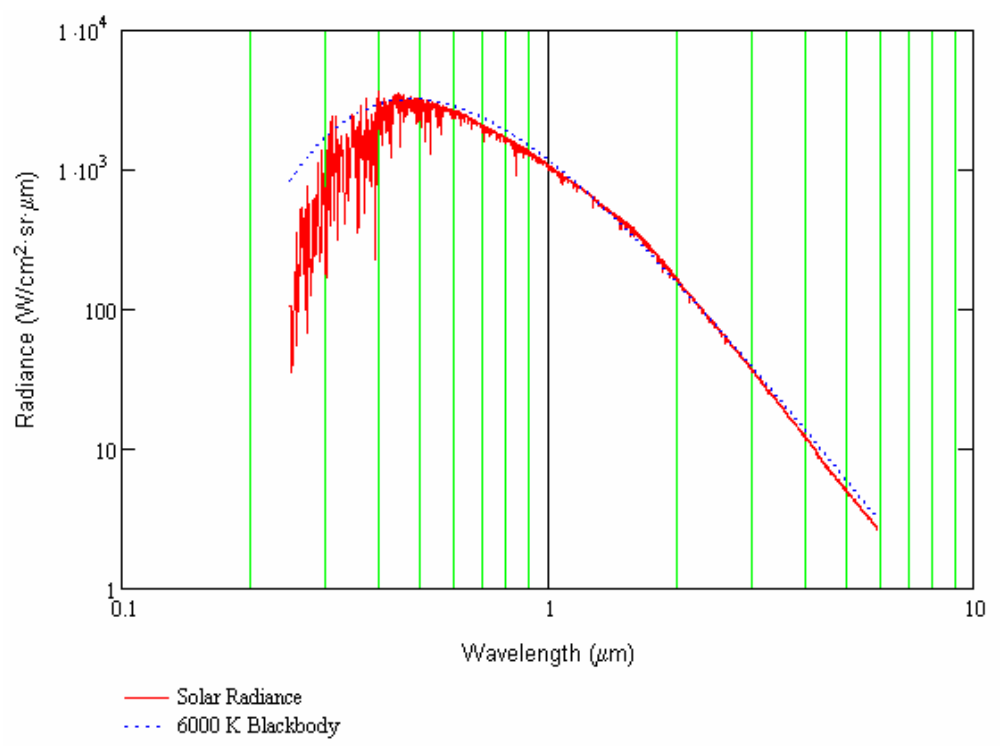

Figure 2. Solar Radiance 
Atmospheric transmittance calculations for a clear-sky standard atmosphere and solar zenith angle of $20^{\circ}$, at Logan, UT, are listed for each SOFIE science band in Table 5. These calculations were used with the solar radiance data shown in Figure 2 to predict sensor response during solar observations.

Table 5: Atmospheric Transmission

\begin{tabular}{|c|c|c|}
\hline Channel & Band / Target & Transmittance \\
\hline \multirow{2}{*}{1} & $1 / \mathrm{O}_{3}$ strong & $2.2768 \times 10^{-4}$ \\
\cline { 2 - 3 } & $2 / \mathrm{O}_{3}$ weak & $4.8443 \times 10^{-1}$ \\
\hline \multirow{2}{*}{2} & $3 /$ Particles strong & $9.8516 \times 10^{-1}$ \\
\cline { 2 - 3 } & $4 /$ Particles weak & $9.9079 \times 10^{-1}$ \\
\hline \multirow{2}{*}{3} & $5 / \mathrm{H}_{2} \mathrm{O}$ weak & $6.2958 \times 10^{-1}$ \\
\cline { 2 - 3 } & $6 / \mathrm{H}_{2} \mathrm{O}$ strong & $4.5285 \times 10^{-5}$ \\
\hline \multirow{2}{*}{4} & $7 / \mathrm{CO}_{2}$ strong & $5.5888 \times 10^{-6}$ \\
\cline { 2 - 3 } & $8 / \mathrm{CO}_{2}$ weak & $3.8243 \times 10^{-1}$ \\
\hline \multirow{2}{*}{5} & $9 /$ Particles strong $^{-1}$ & $3.3414 \times 10^{-1}$ \\
\cline { 2 - 3 } & $10 / \mathrm{Particles} \mathrm{weak}_{1}$ & $5.0245 \times 10^{-1}$ \\
\hline \multirow{2}{*}{6} & $11 / \mathrm{CH}_{4}$ strong & $6.0688 \times 10^{-1}$ \\
\cline { 2 - 3 } & $12 / \mathrm{CH}_{4}$ weak & $9.0109 \times 10^{-1}$ \\
\hline \multirow{2}{*}{7} & $13 / \mathrm{CO}_{2}$ strong & $5.7300 \times 10^{-3}$ \\
\cline { 2 - 3 } & $14 / \mathrm{CO}_{2}$ weak & $8.7377 \times 10^{-1}$ \\
\hline \multirow{2}{*}{8} & $15 / \mathrm{NO}_{\text {weak }}$ & $7.6011 \times 10^{-1}$ \\
\cline { 2 - 3 } & $16 / \mathrm{NO}_{\text {strong }}$ & $3.4663 \times 10^{-1}$ \\
\hline
\end{tabular}

\subsubsection{Focal Plane Test Source}

A radiometric model of an incandescent lamp was implemented to predict and understand focal plane response during steering mirror focal plane bench tests. Important parameters incorporated in this model are shown in Table 6. Visible flux parameters were converted to radiometric quantities using a standard model of human eye response, and total radiant power was then set equal to lamp power to estimate filament effective area. Energy emitted from the lamp filament was assumed to undergo Lambertian scattering by the lamp envelope to arrive at the final lamp radiance.

Table 6: Incandescent Lamp Model Parameters

\begin{tabular}{|c|c|}
\hline Parameter & Value \\
\hline Lamp Power & $200 \mathrm{~W}$ \\
\hline Luminous Flux & $3650 \mathrm{Lumens}$ \\
\hline Filament Temperature & $3000 \mathrm{~K}$ \\
\hline Filament Effective Area & $107 \mathrm{~mm}^{2}$ \\
\hline Filament Effective Emissivity & 0.4 \\
\hline Lamp Envelope transmittance & 0.85 \\
\hline Lamp Envelop Diameter & $2.5 \mathrm{in}$. \\
\hline
\end{tabular}




\section{DESIGN VERIFICATION}

\subsection{Detector Response}

SOFIE detector response was verified by viewing the SEBB source through a collimator to provide full-field coverage of the SOFIE aperture [i]. A range of SEBB temperatures were chosen to provide response over as much of the measurement dynamic range as possible. Measurements were acquired before final installation of a system ND filter in the SOFIE optical path, to provide response higher in the dynamic range than could otherwise be obtained, and again after installation of the ND filter. Sample plots from detector response verification measurements following system ND filter installation are shown in Figure 3. Fitted curves showing ideal linear response and measured non-linear response are shown for comparison.

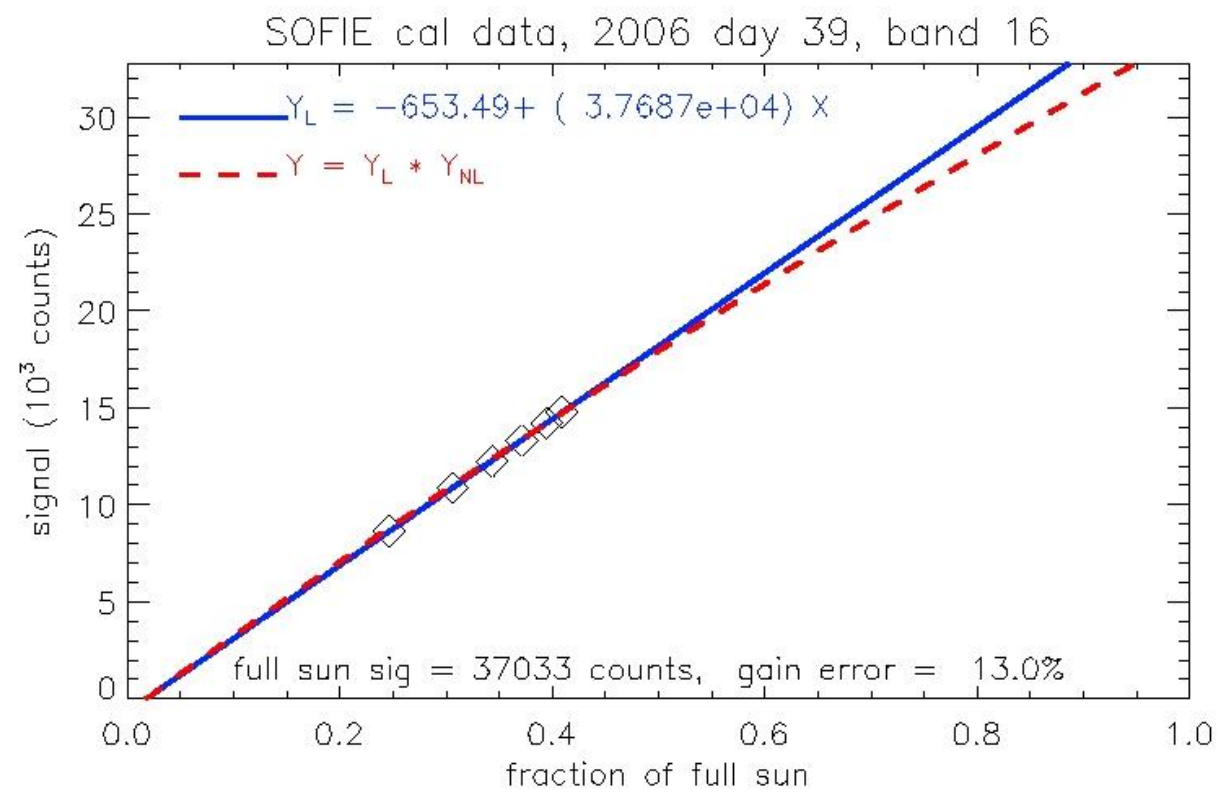

Figure 3. Detector Response Validation Data

Detector response summary data are tabulated in Table 7. SOFIE detector response was designed to achieve full scale response when viewing the Sun from Earth orbit. The gain error shown in Table 7 indicates the additional gain that must be applied to the SOFIE response to achieve this condition. The SOFIE instrument was designed with an adjustable balance attenuation parameter to allow gain changes on the order of those shown.

Table 7. Detector Response Summary

\begin{tabular}{|c|c|c|}
\hline \multirow{2}{*}{ Channel } & Band/Target & Gain Error (\%) \\
\hline \multirow{2}{*}{1} & $1 / \mathrm{O}_{3} \mathrm{~s}$ & 30 \\
\cline { 2 - 3 } & $2 / \mathrm{O}_{3} \mathrm{w}$ & 30 \\
\hline \multirow{2}{*}{2} & $3 / \mathrm{PMC}$ & -4.3 \\
\cline { 2 - 3 } & $4 / \mathrm{PMC}$ & 4.8 \\
\hline \multirow{2}{*}{3} & $5 / \mathrm{H}_{2} \mathrm{O} w$ & 0.0 \\
\cline { 2 - 3 } & $6 / \mathrm{H}_{2} \mathrm{O} \mathrm{s}$ & 8.5 \\
\hline 4 & $7 / \mathrm{CO}_{2} \mathrm{~s}$ & -10.7 \\
\hline
\end{tabular}




\begin{tabular}{|c|c|c|}
\hline \multirow{2}{*}{ Channel } & Band/Target & Gain Error (\%) \\
\hline \multirow{2}{*}{5} & $8 / \mathrm{CO}_{2} \mathrm{w}$ & -5.5 \\
\hline \multirow{2}{*}{6} & $9 / \mathrm{PMC}$ & 0.9 \\
\cline { 2 - 3 } & $10 / \mathrm{PMC}$ & 12.8 \\
\hline \multirow{2}{*}{7} & $11 / \mathrm{CH}_{4} \mathrm{~s}$ & 7.7 \\
\cline { 2 - 3 } & $12 / \mathrm{CH}_{4} \mathrm{w}$ & -12.2 \\
\hline \multirow{2}{*}{8} & $13 / \mathrm{CO}_{2} \mathrm{~s}$ & 1.3 \\
\cline { 2 - 3 } & $14 / \mathrm{CO}_{2} \mathrm{w}$ & 4.9 \\
\cline { 2 - 3 } & $15 / \mathrm{NO}_{\mathrm{w}}$ & 6.3 \\
\cline { 2 - 3 } & $16 / \mathrm{NO} \mathrm{s}$ & 4.8 \\
\hline
\end{tabular}

\subsection{Relative Spectral Response}

In-band RSR measurements were made for each individual spectral band at cold, nominal, and warm science operating temperatures [i]. Band edges determined from nominal temperature RSR measurements are shown in Table 8, compared to requirements. Measured band edges match requirements within a few tens of nanometers or better, the greatest deviation being $49 \mathrm{~nm}$ at the band 14 cut-on edge. Most other band edge deviations are much less. The measured RSR data have been included in measurement simulations, and no science impact is expected as a result of band edge deviations from specification.

Table 8. SOFIE Band Limits

\begin{tabular}{|c|c|c|c|}
\hline \multirow{2}{*}{ Band } & Specification & \multicolumn{2}{|c|}{ Measurement } \\
\cline { 2 - 4 } & $\begin{array}{c}\text { Cut-on }- \text { Cutoff } \\
(\mu \mathbf{m})\end{array}$ & $\begin{array}{c}\text { Cut-on }- \text { Cutoff } \\
(\boldsymbol{\mu m})\end{array}$ & $\begin{array}{c}\text { Cut-on - Cutoff } \\
\text { from requirement } \\
(\boldsymbol{\mu m})\end{array}$ \\
\hline 1 & $0.2857-0.2941$ & $0.2859-0.2972$ & $0.0002-0.0031$ \\
\hline 2 & $0.3226-0.3333$ & $0.3227-0.3375$ & $0.0001-0.0042$ \\
\hline 3 & $0.8475-0.8772$ & $0.8506-0.8831$ & $0.0031-0.0059$ \\
\hline 4 & $1.0101-1.0526$ & $1.015-1.059$ & $0.0051-0.0068$ \\
\hline 5 & $2.427-2.475$ & $2.436-2.488$ & $0.0087-0.0127$ \\
\hline 6 & $2.577-2.632$ & $2.592-2.643$ & $0.0153-0.0115$ \\
\hline 7 & $2.740-2.794$ & $2.758-2.813$ & $0.0178-0.0190$ \\
\hline 8 & $2.907-2.967$ & $2.910-2.968$ & $0.0034-0.0009$ \\
\hline 9 & $3.030-3.091$ & $3.035-3.093$ & $0.0046-0.0025$ \\
\hline 10 & $3.160-3.226$ & $3.152-3.219$ & $-0.0076--0.0066$ \\
\hline 11 & $3.333-3.401$ & $3.345-3.422$ & $0.0121-0.0212$ \\
\hline 12 & $3.472-3.546$ & $3.445-3.514$ & $-0.0272--0.0322$ \\
\hline 13 & $4.255-4.444$ & $4.220-4.428$ & $-0.0352--0.0163$ \\
\hline 14 & $4.630-4.740$ & $4.581-4.712$ & $-0.0491--0.0280$ \\
\hline 15 & $4.951-5.051$ & $4.959-5.053$ & $0.0080-0.0016$ \\
\hline 16 & $5.263-5.376$ & $5.236-5.396$ & $-0.0275-0.0200$ \\
\hline
\end{tabular}

\subsection{Field of View}

SOFIE field of view (FOV) characterization measurements were made to verify the sensor FOV $(1.8$ x 6.0 arcmin nominal) [i]. FOV full-width at half-maximum (FWHM) in azimuth and elevation dimensions is summarized in Table 9. The FOV measured during ground calibration is consistent with the design specification used in the radiometric model. 
Table 9. SOFIE Field-Of-View

\begin{tabular}{|c|c|c|c|c|c|}
\hline \multirow[b]{2}{*}{ Chan. } & \multirow{2}{*}{$\begin{array}{l}\text { Band / } \\
\text { Target }\end{array}$} & \multicolumn{2}{|c|}{ Elevation Dimension } & \multicolumn{2}{|c|}{ Azimuth Dimension } \\
\hline & & $\begin{array}{l}\text { Mean FWHM } \\
\text { (arcmin) }\end{array}$ & $\begin{array}{l}\text { Mean FWHM } \\
\text { (arcmin) }\end{array}$ & $\begin{array}{c}\text { Mean FWHM } \\
\text { (arcmin) }\end{array}$ & $\begin{array}{l}\text { Uncertainty } \\
\text { (arcmin) }\end{array}$ \\
\hline \multirow{2}{*}{1} & $1 / \mathrm{O}_{3} \mathrm{~s}$ & 1.43 & 0.15 & 7.11 & 0.50 \\
\hline & $2 / \mathrm{O}_{3} \mathrm{~W}$ & 1.64 & 0.15 & 8.87 & 0.50 \\
\hline \multirow{2}{*}{2} & $3 / \mathrm{PMC}$ & 1.83 & 0.09 & 5.35 & 0.42 \\
\hline & 4 / PMC & 1.82 & 0.09 & 5.35 & 0.83 \\
\hline \multirow{2}{*}{3} & $5 / \mathrm{H}_{2} \mathrm{O} w$ & 1.91 & 0.06 & 4.14 & 0.63 \\
\hline & $6 / \mathrm{H}_{2} \mathrm{O} \mathrm{s}$ & 1.98 & 0.07 & 4.81 & 0.40 \\
\hline \multirow{2}{*}{4} & $7 / \mathrm{CO}_{2} \mathrm{~s}$ & 2.08 & 0.09 & 4.91 & 0.50 \\
\hline & $8 / \mathrm{CO}_{2} \mathrm{~W}$ & 2.05 & 0.12 & 5.57 & 1.06 \\
\hline \multirow{2}{*}{5} & 9 / PMC & 1.99 & 0.07 & 3.73 & 0.86 \\
\hline & $10 /$ PMC & 1.91 & 0.08 & 5.12 & 0.45 \\
\hline \multirow{2}{*}{6} & $11 / \mathrm{CH}_{4} \mathrm{~s}$ & 2.09 & 0.11 & 5.48 & 0.36 \\
\hline & $12 / \mathrm{CH}_{4} \mathrm{~W}$ & 1.90 & 0.13 & 5.53 & 0.42 \\
\hline \multirow{2}{*}{7} & $13 / \mathrm{CO}_{2} \mathrm{~s}$ & 1.96 & 0.11 & 5.81 & 0.44 \\
\hline & $14 / \mathrm{CO}_{2} \mathrm{~W}$ & 1.94 & 0.10 & 5.57 & 0.45 \\
\hline \multirow{2}{*}{8} & $15 / \mathrm{NO} w$ & 1.96 & 0.10 & 5.34 & 0.57 \\
\hline & $16 / \mathrm{NO} s$ & 1.95 & 0.12 & 4.73 & 0.66 \\
\hline
\end{tabular}

\subsection{Detector Nonlinearity}

Detector nonlinearity is generally hard to predict and therefore difficult to model effectively. SOFIE radiometric models were therefore implemented under the assumption of ideal linear response. However, calibration testing revealed significant nonlinearity, which was traced to nonlinear behavior in $\mathrm{HgCdTe}$ detectors when the irradiance (flux per area) on the detectors exceeds a certain level [ii]. SOFIE detector nonlinearity was then characterized using a small attenuator method [i]. Detector nonlinearity is summarized in Table 10.

Table 10. SOFIE Nonlinearity Correction Constants

\begin{tabular}{|c|c|c|c|c|c|}
\hline Channel & $\begin{array}{c}\text { Band / } \\
\text { Target* }\end{array}$ & $\begin{array}{c}\text { Center } \lambda \\
(\mu \mathrm{m})\end{array}$ & Detector & $C_{N L}($ counts-1) & $\begin{array}{l}\text { Nonlinearity }(\%) \text { at } 2^{15} \\
\text { counts / uncertainty }(\%)\end{array}$ \\
\hline \multirow{2}{*}{1} & $1 / \mathrm{O}_{3} \mathrm{~s}$ & 0.290 & \multirow{2}{*}{$\mathrm{SiC}, \mathrm{PV}$} & 0 & 0 \\
\hline & $2 / \mathrm{O}_{3} \mathrm{w}$ & 0.328 & & 0 & 0 \\
\hline \multirow{2}{*}{2} & $3 / \mathrm{PMC}$ s & 0.862 & \multirow{2}{*}{$\mathrm{Ge}, \mathrm{PV}$} & 0 & 0 \\
\hline & 4 / PMC w & 1.03 & & 0 & 0 \\
\hline \multirow{2}{*}{3} & $5 / \mathrm{H}_{2} \mathrm{O} w$ & 2.45 & \multirow{2}{*}{$\mathrm{HgCdTe}, \mathrm{PC}$} & $1.68 \times 10-6$ & $5.5 / 0.3$ \\
\hline & $6 / \mathrm{H}_{2} \mathrm{O} \mathrm{s}$ & 2.60 & & $1.46 \times 10-6$ & $4.8 / 0.3$ \\
\hline \multirow{2}{*}{4} & $7 / \mathrm{CO}_{2} \mathrm{~s}$ & 2.77 & \multirow{2}{*}{$\mathrm{HgCdTe}, \mathrm{PC}$} & $8.91 \times 10-6$ & $29.2 / 0.2$ \\
\hline & $8 / \mathrm{CO}_{2} \mathrm{~W}$ & 2.94 & & $7.94 \times 10-6$ & 26.00 .2 \\
\hline \multirow{2}{*}{5} & $9 / \mathrm{PMC} \mathrm{s}$ & 3.06 & \multirow{2}{*}{$\mathrm{HgCdTe}, \mathrm{PC}$} & $6.63 \times 10-7$ & $2.2 / 0.2$ \\
\hline & $10 / \mathrm{PMC} w$ & 3.19 & & $1.47 \times 10-6$ & $4.8 / 0.2$ \\
\hline \multirow{2}{*}{6} & $11 / \mathrm{CH}_{4} \mathrm{~s}$ & 3.37 & \multirow{2}{*}{$\mathrm{HgCdTe}, \mathrm{PC}$} & $1.46 \times 10-6$ & $4.8 / 0.2$ \\
\hline & $12 / \mathrm{CH}_{4} \mathrm{~W}$ & 3.51 & & $2.23 \times 10-6$ & $7.3 / 0.2$ \\
\hline
\end{tabular}




\begin{tabular}{|c|c|c|c|c|c|}
\hline Channel & $\begin{array}{c}\text { Band / } \\
\text { Target* }\end{array}$ & $\begin{array}{c}\text { Center } \boldsymbol{\lambda} \\
(\boldsymbol{\mu} \mathbf{m})\end{array}$ & \multirow{2}{*}{ Detector } & \multirow{2}{*}{$\boldsymbol{C}_{\boldsymbol{N}}$ (counts-1) } & $\begin{array}{c}\text { Nonlinearity (\%) at 25 } \\
\text { counts / uncertainty (\%) }\end{array}$ \\
\hline \multirow{2}{*}{7} & $13 / \mathrm{CO}_{2} \mathrm{~s}$ & 4.25 & \multirow{2}{*}{ HgCdTe, PC } & $4.83 \times 10-6$ & $15.8 / 0.7$ \\
\cline { 2 - 3 } & $14 / \mathrm{CO}_{2} \mathrm{~W}$ & 4.63 & & $3.20 \times 10-6$ & $10.5 / 0.9$ \\
\hline \multirow{2}{*}{8} & $15 / \mathrm{NO} \mathrm{w}$ & 4.98 & \multirow{2}{*}{$\mathrm{HgCdTe}, \mathrm{PC}$} & $1.75 \times 10-6$ & $5.7 / 0.2$ \\
\cline { 2 - 3 } & $16 / \mathrm{NO} \mathrm{s}$ & 5.32 & & $2.26 \times 10-6$ & $7.4 / 0.3$ \\
\hline
\end{tabular}

\subsection{Steering Mirror Focal Plane Response}

Preliminary sun sensor testing was conducted using the incandescent lamp. A sample sun sensor image of the lamp from this testing is shown in Figure 4. The lamp filament is visible, as well as some structure in the lamp envelope. Because of the preliminary nature of the hardware during this phase of testing, numerical response data for lamp source measurements could not be acquired for comparison to the model, although the response did seem consistent with predictions.

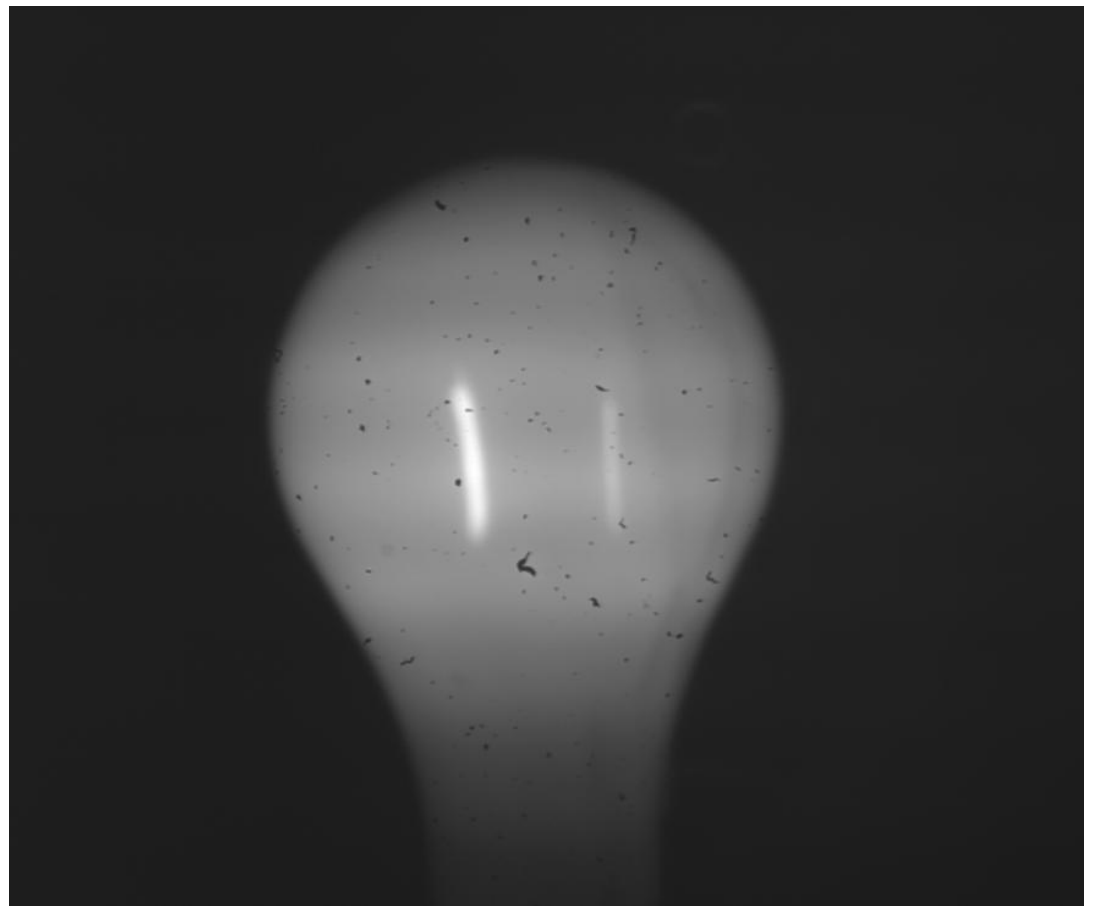

Figure 4. Sun Sensor Image of Lamp Source

Additional sun sensor testing was conducted while observing the sun directly. A heliostat system was used to maintain a stable sun reflection on the sun sensor hardware. Predicted response levels for Sun observations are shown in Figure 5, with measured response levels for comparison. The predictions are consistent with the measured data, although the predicted results assume linear response, and the measured response obviously shows some roll-off at higher response levels. Based on the outcome of these tests, the sun sensor bandpass filter was replaced with a filter covering the range $688-713 \mathrm{~nm}$, to provide some additional response to meet sampling speed requirements. 


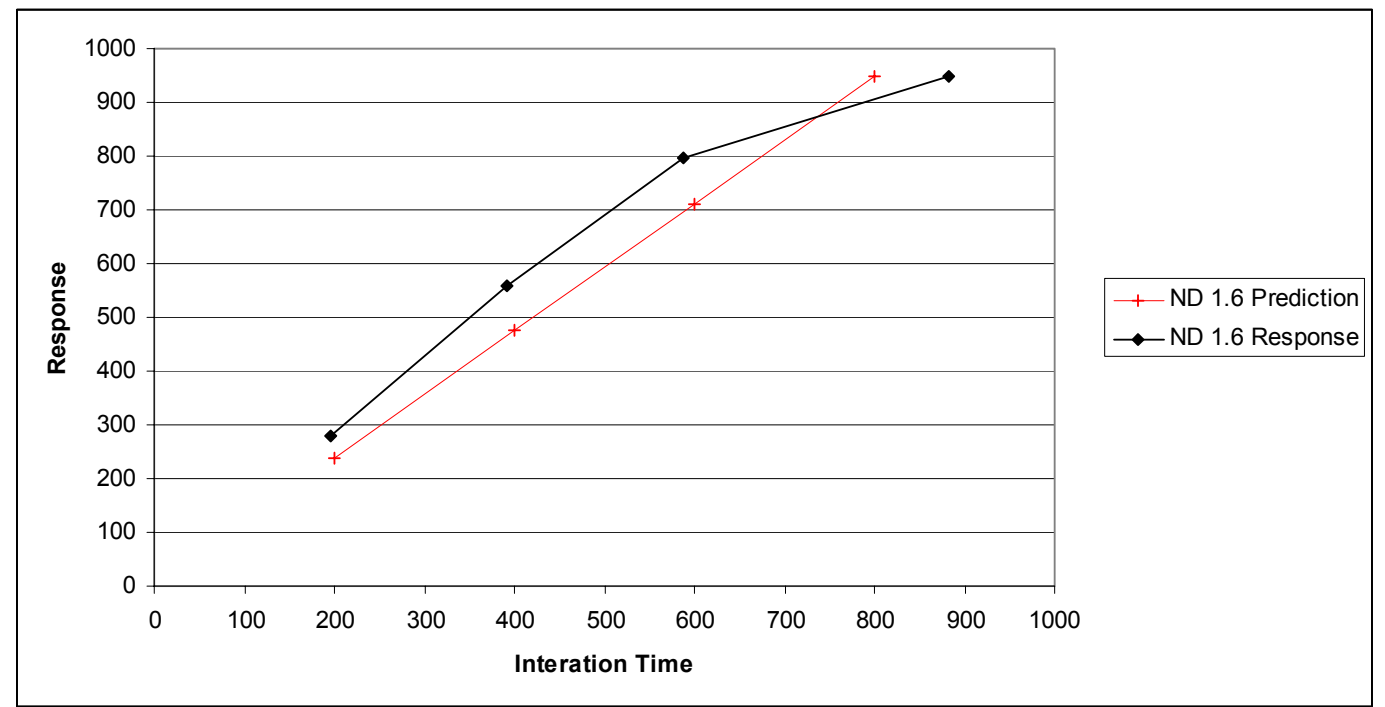

Figure 5. Sun Sensor Response vs. Prediction

\section{SUMMARY AND CONCLUSION}

Theoretical models of the SOFIE science detectors and steering mirror focal plane were important tools used during instrument design and fabrication to understand instrument performance, and to develop calibration plans and methods. During instrument test and calibration, measurements were made that confirmed parameters used in the radiometric models, or revealed model shortcomings and contributed to an improved understanding of the SOFIE instrument. Detailed radiometric modeling forms an integral part of the design process at the Space Dynamics Laboratory, and the success of this approach has again been demonstrated by the SOFIE instrument. The SOFIE instrument is ready to go to work.

i. Hansen, S., C. Fish, D. Romrell, L. Gordley, and M. Hervig, "SOFIE Instrument Ground Calibration,” Proc. of SPIE $6297,2006$. ii. Theocharous, E., J. Ishii and N. P. Fox, “Absolute linearity measurements on HgCdTe detectors in the infrared region," Applied Optics Vol. 43, No. 21, 4182-4188, July 2004. 